\title{
Generalized Hyers-Ulam Stability of Quadratic Functional Inequality
}

\author{
Hark-Mahn Kim, Kil-Woung Jun, and Eunyoung Son \\ Department of Mathematics, Chungnam National University, 79 Daehangno, Yuseong-Gu, Daejeon 305-764, Republic of Korea \\ Correspondence should be addressed to Eunyoung Son; sey8405@hanmail.net
}

Received 17 June 2013; Revised 7 October 2013; Accepted 30 October 2013

Academic Editor: Krzysztof Ciepliński

Copyright ( 2013 Hark-Mahn Kim et al. This is an open access article distributed under the Creative Commons Attribution License, which permits unrestricted use, distribution, and reproduction in any medium, provided the original work is properly cited.

We establish the general solution of the functional inequality $\|f(x-y)+f(y-z)+f(x-z)-3 f(x)-3 f(y)-3 f(z)\| \leq\|f(x+y+z)\|$ and then investigate the generalized Hyers-Ulam stability of this inequality in Banach spaces and in non-Archimedean Banach spaces.

\section{Introduction}

The stability problem of functional equations originated from a question of Ulam [1] in 1940, concerning the stability of group homomorphisms.

We are given a group $G$ and a metric group $G^{\prime}$ with metric $\rho(\cdot, \cdot)$. Given $\epsilon>0$, does there exist a number $\delta>0$ such that if $f: G \rightarrow G^{\prime}$ satisfies $\rho(f(x y), f(x) f(y))<\delta$ for all $x, y \in G$, then a homomorphism $h: G \rightarrow G^{\prime}$ exists with $\rho(f(x), h(x))<\epsilon$ for all $x \in G$ ?

Hyers [2] gave a first affirmative partial answer to the question of Ulam for Banach spaces by using, so called, direct method. Hyers' theorem was generalized by Aoki [3] for additive mappings and by Rassias [4] for linear mappings by considering an unbounded Cauchy difference. A generalization of the Rassias' theorem was obtained by Găvruta [5] by replacing the unbounded Cauchy difference by a general control function in the spirit of the Rassias' approach.

Let $E_{1}$ and $E_{2}$ be vector spaces. A mapping $f: E_{1} \rightarrow E_{2}$ is called quadratic if and only if it is a solution of the quadratic functional equation:

$$
f(x+y)+f(x-y)=2 f(x)+2 f(y)
$$

for all $x, y \in X$. It is well known that a function $f$ between real vector spaces is quadratic if and only if there exists a unique symmetric biadditive function $B$ such that $f(x)=B(x, x)$ for all $x$, where the mapping $B$ is given by $B(x, y)=(1 / 4)[f(x+$ $y)-f(x-y)]([6,7])$. The Hyers-Ulam stability of the quadratic functional equation (1) was first proved by Skof [8] for functions $f: E_{1} \rightarrow E_{2}$, where $E_{1}$ is a normed space and $E_{2}$ is a Banach space. Cholewa [9] noticed that Skof's theorem is also valid if $E_{1}$ is replaced by an abelian group. In 1992, Czerwik [10] proved the generalized Hyers-Ulam stability of quadratic functional equation (1) in the spirit of Rassias approach by using direct method [11].

The generalized Hyers-Ulam stability of the above quadratic functional equation and of two functional equations of quadratic type was obtained by Cădariu and Radu [12, 13] by using fixed point method (see also $[14,15]$ ).

Gilányi [16] and Rätz [17] proved that for a function $f$ : $G \rightarrow E$ mapping from an abelian group $G$ divisible by 2 into an inner product space $E$, the functional inequality

$$
\left\|2 f(x)+2 f(y)-f\left(x y^{-1}\right)\right\| \leq\|f(x y)\| \quad(x, y \in G)
$$

implies the Jordan-von Neumann functional equation

$$
2 f(x)+2 f(y)=f(x y)+f\left(x y^{-1}\right) \quad(x, y \in G) .
$$

Fechner [18] and Gilányi [19] have proved the generalized Hyers-Ulam stability of the functional inequality (2). Park et al. [20] have investigated the generalized Hyers-Ulam stability of functional inequalities associated with Jordanvon Neumann type additive functional equations, and Kim et al. [21] have proved the generalized Hyers-Ulam stability of Jensen functional inequality in $p$-Banach spaces. The stability 
problems of several functional equations and inequalities have been extensively investigated by a number of authors and there are many interesting results concerning the stability of various functional equations and inequalities [6, 22].

In 2001, Bae and Kim [23] investigated the Hyers-Ulam stability of the quadratic functional equation

$$
\begin{aligned}
f(x+y+z) & +f(x-y)+f(y-z) \\
& +f(x-z)=3 f(x)+3 f(y)+3 f(z)
\end{aligned}
$$

which is equivalent to the original quadratic functional equation (1). Now, let us turn our attention to investigate the generalized stability problem of the following quadratic functional inequality:

$$
\begin{aligned}
& \|f(x-y)+f(y-z)+f(x-z)-3 f(x)-3 f(y)-3 f(z)\| \\
& \quad \leq\|f(x+y+z)\| .
\end{aligned}
$$

In this paper, we make an attempt to establish the generalized Hyers-Ulam stability of a new quadratic functional inequality (5) by using fixed point method and direct method. In Section 2, we establish the general solution of the functional inequality (5). And then we prove the generalized Hyers-Ulam stability of the functional inequality (5) in Banach spaces by using fixed point method. In Section 3, we verify the generalized Hyers-Ulam stability of the functional inequality (5) in Banach spaces by using direct method. In Section 4, we investigate the generalized Hyers-Ulam stability of the functional inequality (5) in non-Archimedean Banach spaces by using fixed point method. In Section 5, we prove the generalized Hyers-Ulam stability of the functional inequality (5) in non-Archimedean Banach spaces by using direct method.

\section{Stability of (5) by Fixed Point Method}

In [24], Rassias introduced the following equality:

$$
\sum_{i, j=1}^{n}\left\|x_{i}-x_{j}\right\|^{2}=2 n \sum_{i=1}^{n}\left\|x_{i}\right\|^{2}, \quad \sum_{i=1}^{n} x_{i}=0
$$

for a fixed integer $n \geq 3$. Let $V, W$ be real vector space. It has been proved that if a mapping $f: V \rightarrow W$ satisfies

$$
\sum_{i, j=1}^{n} f\left(x_{i}-x_{j}\right)=2 n \sum_{i=1}^{n} f\left(x_{i}\right),
$$

for all $x_{1}, \ldots, x_{n} \in V$ with $\sum_{i=1}^{n} x_{i}=0$, then the mapping $f$ is realized as the sum of an additive mapping and a quadratic mapping [25]. Park [25] and Jang et al. [26] have proved the generalized Hyers-Ulam stability of the functional equation (7). In particular, if $n=3$ and even function $f$ satisfies (7), then it is easy to see that $f$ satisfies the equation

$$
\begin{array}{r}
f(x-y)+f(2 x+y)+f(x+2 y) \\
=3 f(x)+3 f(y)+3 f(x+y)
\end{array}
$$

for all $x, y \in X$. Thus, we first consider the general solution of functional equation (8) to verify the general solution of functional inequality (5).

Lemma 1. Let both $X$ and $Y$ be vector spaces. A function $f$ : $X \rightarrow Y$ satisfies (8) if and only if $f$ is quadratic.

Proof. If we put $x=y=0$ in (8), then we have $f(0)=0$. Letting $y=0$ in (8), we have $f(2 x)=4 f(x)$ for all $x \in X$. Similarly, we can easily show that $f(3 x)=9 f(x)$ and $f(5 x)=$ $25 f(x)$ for all $x \in X$. Replacing $y$ by $-x$ in $(8)$, we get $f(-x)=$ $f(x)$ for all $x \in X$. Substituting $x+y$ for $y$ in (8), we obtain

$$
\begin{aligned}
f(y) & +f(3 x+y)+f(3 x+2 y) \\
& =3 f(x)+3 f(x+y)+3 f(2 x+y)
\end{aligned}
$$

for all $x, y \in X$. Switching $x$ with $y$ in (9) yields

$$
\begin{aligned}
f(x) & +f(x+3 y)+f(2 x+3 y) \\
& =3 f(y)+3 f(x+y)+3 f(x+2 y)
\end{aligned}
$$

for all $x, y \in X$. Adding (9) to (10) and using (8), we arrive at

$$
\begin{array}{r}
f(3 x+2 y)+f(2 x+3 y)+f(3 x+y)+f(x+3 y) \\
=11 f(x)+11 f(y)+15 f(x+y)-3 f(x-y)
\end{array}
$$

for all $x, y \in X$. Letting $y:=2 y$ in (8), we obtain

$$
\begin{aligned}
f(x-2 y)+4 f(x+y)+f(x+4 y) \\
=3 f(x)+12 f(y)+3 f(x+2 y)
\end{aligned}
$$

for all $x, y \in X$. Switching $x$ with $y$ in (12) and using the evenness of $f$, one gets

$$
\begin{aligned}
& f(2 x-y)+4 f(x+y)+f(4 x+y) \\
& =12 f(x)+3 f(y)+3 f(2 x+y)
\end{aligned}
$$

for all $x, y \in X$. Adding (12) to (13) and using (8), we have

$$
\begin{aligned}
f(x+4 y) & +f(4 x+y) \\
& =21 f(x)+21 f(y)+2 f(x+y)-6 f(x-y)
\end{aligned}
$$

for all $x, y \in X$. Putting $x:=x-y$ and $y:=2 x+3 y$ in (8), we get

$$
\begin{aligned}
f(x+4 y) & +f(4 x+y) \\
= & 3 f(x-y)+3 f(2 x+3 y) \\
& +3 f(3 x+2 y)-25 f(x+y)
\end{aligned}
$$

for all $x, y \in X$. From (14) and (15), it follows that

$$
\begin{aligned}
f(3 x & +2 y)+f(2 x+3 y) \\
& =9 f(x+y)+7 f(x)+7 f(y)-3 f(x-y)
\end{aligned}
$$


for all $x, y \in X$. By (11) and (16), we obtain

$$
f(3 x+y)+f(x+3 y)=6 f(x+y)+4 f(x)+4 f(y)
$$

for all $x, y \in X$. Letting $x:=(x+y) / 2$ and $y:=(x-y) / 2$ in (17), we have

$$
f(2 x+y)+f(2 x-y)=6 f(x)+f(x+y)+f(x-y)
$$

for all $x, y \in X$. Interchanging $x$ for $y$ in (18) and using the evenness of $f$, one gets

$$
f(x+2 y)+f(x-2 y)=6 f(y)+f(x+y)+f(x-y)
$$

for all $x, y \in X$. Substituting $2 y$ for $y$ in (18), we get by virtue of (19)

$$
\begin{aligned}
4 f(x & +y)+4 f(x-y) \\
& =6 f(x)+f(x+2 y)+f(x-2 y) \\
& =6 f(x)+6 f(y)+f(x+y)+f(x-y),
\end{aligned}
$$

which yields

$$
f(x+y)+f(x-y)=2 f(x)+2 f(y)
$$

for all $x, y \in X$. Thus, the mapping $f$ is quadratic.

The proof of the converse is trivial.

Now, we present the general solution of the functional inequality (5) by using Lemma 1.

Lemma 2. Let both $X$ and $Y$ be vector spaces. A mapping $f$ : $X \rightarrow Y$ satisfies the functional inequality (5) for all $x, y, z \in X$ if and only if $f$ is quadratic.

Proof. Let $f$ satisfy the functional inequality (5). If we replace $x, y$, and $z$ in (5) by 0 , then we have $f(0)=0$. Replacing $z$ by $-x-y$ in (5), we obtain

$$
\begin{aligned}
f(x-y) & +f(x+2 y)+f(2 x+y) \\
& -3 f(x)-3 f(y)-3 f(-x-y)=0
\end{aligned}
$$

for all $x, y \in X$. Letting $y:=-x$ and $z:=0$ in (5), we get

$$
f(2 x)-2 f(x)-2 f(-x)=0
$$

for all $x \in X$. Putting $y:=0$ in (22) yields

$$
f(2 x)-f(x)-3 f(-x)=0
$$

for all $x \in X$. From (23) and (24), we have $f(-x)=f(x)$ for all $x \in X$. Thus, it follows from (22) that

$$
\begin{array}{r}
f(x-y)+f(x+2 y)+f(2 x+y) \\
=3 f(x)+3 f(y)+3 f(x+y)
\end{array}
$$

for all $x, y \in X$. So $f$ is quadratic by Lemma 1 .

The proof of the converse is trivial.
Let $X$ be a set. A function $d: X \times X \rightarrow[0, \infty]$ is called a generalized metric on $X$ if $d$ satisfies the following:

(1) $d(x, y)=0$ if and only if $x=y$;

(2) $d(x, y)=d(y, x)$ for all $x, y \in X$;

(3) $d(x, z) \leq d(x, y)+d(y, z)$ for all $x, y, z \in X$.

Before taking up the main subject, we recall the fixed point theorem from [14].

Theorem 3 (see [14]). Let $(X, d)$ be a complete generalized metric space and let $J: X \rightarrow X$ be a strictly contractive mapping with Lipschitz constant $L<1$. Then for each given element $x \in X$, either

$$
d\left(J^{n} x, J^{n+1} x\right)=\infty
$$

for all nonnegative integers $n$ or there exists a positive integer $n_{0}$ such that

(1) $d\left(J^{n} x, J^{n+1} x\right)<\infty$, for all $n \geq n_{0}$;

(2) the sequence $\left\{J^{n} x\right\}$ converges to a fixed point $y^{*}$ of $J$;

(3) $y^{*}$ is the unique fixed point of $J$ in the set $Y=\{y \in X \mid$ $\left.d\left(J^{n_{0}} x, y\right)<\infty\right\}$

(4) $d\left(y, y^{*}\right) \leq(1 /(1-L)) d(y$, Jy) for all $y \in Y$.

In this part, let $X$ be a normed space and $Y$ a Banach space.

Theorem 4. Suppose that a mapping $f: X \rightarrow Y$ with $f(0)=$ 0 satisfies the functional inequality

$$
\begin{aligned}
& \|f(x-y)+f(y-z)+f(x-z)-3 f(x)-3 f(y)-3 f(z)\| \\
& \leq\|f(x+y+z)\|+\varphi(x, y, z)
\end{aligned}
$$

for all $x, y, z \in X$ and that there exists a constant $L$ with $0<$ $L<1$ for which the perturbing function $\varphi: X^{3} \rightarrow[0, \infty)$ satisfies

$$
\begin{gathered}
\varphi(2 x, 2 y, 2 z) \leq 4 L \varphi(x, y, z), \\
\left(\varphi\left(\frac{x}{2}, \frac{y}{2}, \frac{z}{2}\right) \leq \frac{L}{4} \varphi(x, y, z), \text { resp. }\right)
\end{gathered}
$$

for all $x, y, z \in X$. Then, there exists a unique quadratic mapping $Q: X \rightarrow Y$ given by $Q(x)=\lim _{m \rightarrow \infty}\left(1 / 4^{m}\right) f\left(2^{m} x\right)$ $\left(Q(x)=\lim _{m \rightarrow \infty} 4^{m} f\left(x / 2^{m}\right)\right.$, resp. $)$ such that

$$
\begin{aligned}
& \|f(x)-Q(x)\| \leq \frac{1}{4-4 L}\{3 \varphi(x,-x, 0)+2 \varphi(x, 0,-x)\}, \\
& (\|f(x)-Q(x)\| \\
& \left.\quad \leq \frac{L}{4-4 L}\{3 \varphi(x,-x, 0)+2 \varphi(x, 0,-x)\}, \text { resp. }\right)
\end{aligned}
$$

for all $x \in X$. 
Proof. Replacing $z$ by $-x-y$ in (27), we obtain

$$
\begin{aligned}
& \| f(x-y)+f(x+2 y)+f(2 x+y) \\
& \quad-3 f(x)-3 f(y)-3 f(-x-y) \| \leq \varphi(x, y,-x-y)
\end{aligned}
$$

for all $x, y \in X$. Letting $y=-x$ and $z=0$ in (27), we get

$$
\|f(2 x)-2 f(x)-2 f(-x)\| \leq \varphi(x,-x, 0)
$$

for all $x \in X$. Putting $y=0$ in (30) yields

$$
\|f(2 x)-f(x)-3 f(-x)\| \leq \varphi(x, 0,-x)
$$

for all $x \in X$. From (31) and (32), it follows that

$$
\|f(2 x)-4 f(x)\| \leq 3 \varphi(x,-x, 0)+2 \varphi(x, 0,-x): \equiv \Phi(x)
$$

and so

$$
\left\|f(x)-\frac{1}{4} f(2 x)\right\| \leq \frac{1}{4} \Phi(x)
$$

for all $x \in X$.

Consider the set of mappings

$$
S:=\{h: X \longrightarrow Y \mid h(0)=0\}
$$

and introduce the generalized metric on $S$ :

$d(g, h)$

$$
=\inf \{\mu \in[0, \infty):\|g(x)-h(x)\| \leq \mu \Phi(x), \forall x \in X\},
$$

where, as usual, inf $\emptyset=+\infty$. It is easy to show that $(S, d)$ is a complete generalized metric space (see the proof of Theorem 3.1 of [27]).

Now we consider a linear mapping $J: S \rightarrow S$ such that

$$
J g(x):=\frac{1}{4} g(2 x)
$$

for all $x \in X$. Then it is well-known that $J$ is a strictly contractive mapping with Lipschitz constant $L$, and it follows from (34) that $d(f, J f) \leq 1 / 4$. By Theorem 3 , there exists a mapping $Q: X \rightarrow Y$ satisfying the following.

(1) $Q$ is a fixed point of $J$, that is, $Q(2 x)=4 Q(x)$ for all $x \in X$. The mapping $Q$ is a unique fixed point of $J$ in the set $M=\{g \in S: d(f, g)<\infty\}$. This implies that $Q$ is a unique fixed point of $J$ such that there exists a $\mu \in(0, \infty)$ satisfying

$$
\|f(x)-Q(x)\| \leq \mu \Phi(x),
$$

for all $x \in X$.

(2) $d\left(J^{m} f, Q\right) \rightarrow 0$ as $m \rightarrow \infty$. This implies the equality $Q(x)=\lim _{m \rightarrow \infty}\left(1 / 4^{m}\right) f\left(2^{m} x\right)$ for all $x \in X$.
(3) $d(f, Q) \leq(1 /(1-L)) d(f, J f) \leq 1 /(4-4 L)$, which implies that the inequality (29) holds.

Now, we show that the mapping $Q$ is quadratic. It follows from (27) and (28) that

$$
\begin{gathered}
\|Q(x-y)+Q(y-z)+Q(x-z)-3 Q(x)-3 Q(y)-3 Q(z)\| \\
-\|Q(x+y+z)\| \leq \lim _{m \rightarrow \infty} \frac{1}{4^{m}} \varphi\left(2^{m} x, 2^{m} y, 2^{m} z\right) \\
\leq \lim _{m \rightarrow \infty} L^{m} \varphi(x, y, z)=0
\end{gathered}
$$

for all $x, y, z \in X$. Thus, it follows from Lemma 2 that the mapping $Q: X \rightarrow Y$ is quadratic, as desired.

We obtain the following corollary concerning the stability for approximate mappings controlled by a sum of powers of norms.

Corollary 5. Let $\theta \geq 0$ be a real number and $p$ a positive real number with $p \neq 2$. If a mapping $f: X \rightarrow Y$ with $f(0)=0$ satisfies the inequality

$$
\begin{gathered}
\|f(x-y)+f(y-z)+f(x-z)-3 f(x)-3 f(y)-3 f(z)\| \\
\leq\|f(x+y+z)\|+\theta\left(\|x\|^{p}+\|y\|^{p}+\|z\|^{p}\right)
\end{gathered}
$$

for all $x, y, z \in X$, then we can find a unique quadratic mapping $Q: X \rightarrow Y$ satisfying the inequality

$$
\|f(x)-Q(x)\| \leq \frac{10 \theta}{\left|4-2^{p}\right|}\|x\|^{p}
$$

for all $x \in X$.

\section{Stability of (5) by Direct Method}

We now investigate stability problem of the quadratic functional inequality (5) with perturbed control function $\varphi$. In this section, let $X$ be a normed space and $Y$ a Banach space.

Theorem 6. Suppose that a mapping $f: X \rightarrow Y$ with $f(0)=$ 0 satisfies the functional inequality (27) and that the perturbing function $\varphi: X^{3} \rightarrow[0, \infty)$ satisfies

$$
\begin{gathered}
\sum_{i=0}^{\infty} \frac{1}{4^{i}} \varphi\left(2^{i} x, 2^{i} y, 2^{i} z\right)<\infty, \\
\left(\sum_{i=1}^{\infty} 4^{i} \varphi\left(\frac{x}{2^{i}}, \frac{y}{2^{i}}, \frac{z}{2^{i}}\right)<\infty, \text { resp. }\right)
\end{gathered}
$$


for all $x, y, z \in X$. Then, there exists a unique quadratic mapping $Q: X \rightarrow Y$ defined by $Q(x)=\lim _{m \rightarrow \infty}\left(1 / 4^{m}\right) f\left(2^{m} x\right)$ $\left(Q(x)=\lim _{m \rightarrow \infty} 4^{m} f\left(x / 2^{m}\right)\right.$, resp. $)$ such that

$$
\begin{aligned}
& \|f(x)-Q(x)\| \\
& \leq \frac{1}{4} \sum_{i=0}^{\infty} \frac{1}{4^{i}}\left\{3 \varphi\left(2^{i} x,-2^{i} x, 0\right)+2 \varphi\left(2^{i} x, 0,-2^{i} x\right)\right\}, \\
& \left(\|f(x)-Q(x)\| \leq \frac{1}{4} \sum_{i=1}^{\infty} 4^{i}\left\{3 \varphi\left(\frac{x}{2^{i}}, \frac{-x}{2^{i}}, 0\right)\right.\right. \\
& \left.\left.+2 \varphi\left(\frac{x}{2^{i}}, 0, \frac{-x}{2^{i}}\right)\right\}, \text { resp. }\right)
\end{aligned}
$$

for all $x \in X$.

Proof. It follows from (33) that

$$
\left\|f(x)-\frac{1}{4} f(2 x)\right\| \leq \frac{1}{4}\{3 \varphi(x,-x, 0)+2 \varphi(x, 0,-x)\}
$$

for all $x \in X$. Therefore, we prove from inequality (44) that for any integers $m$ and $l$ with $m>l \geq 0$

$$
\begin{aligned}
& \left\|\frac{f\left(2^{l} x\right)}{4^{l}}-\frac{f\left(2^{m} x\right)}{4^{m}}\right\| \\
& \quad \leq \sum_{i=l}^{m-1} \frac{1}{4^{i}}\left\|f\left(2^{i} x\right)-\frac{1}{4} f\left(2^{i+1} x\right)\right\| \\
& \quad \leq \frac{1}{4} \sum_{i=l}^{m-1} \frac{1}{4^{i}}\left\{3 \varphi\left(2^{i} x,-2^{i} x, 0\right)+2 \varphi\left(2^{i} x, 0,-2^{i} x\right)\right\}
\end{aligned}
$$

for all $x \in X$. Since the right-hand side of (45) tends to zero as $l \rightarrow \infty$, we obtain that the sequence $\left\{f\left(2^{m} x\right) / 4^{m}\right\}$ is Cauchy for all $x \in X$. Because of the fact that $Y$ is complete, it follows that the sequence $\left\{f\left(2^{m} x\right) / 4^{m}\right\}$ converges in $Y$. Therefore, we can define a mapping $Q: X \rightarrow Y$ as

$$
Q(x)=\lim _{m \rightarrow \infty} \frac{f\left(2^{m} x\right)}{4^{m}}, \quad x \in X .
$$

Moreover, letting $l=0$ and taking $m \rightarrow \infty$ in (45), we get the desired inequality (43).

It follows from (27) and (42) that

$$
\begin{array}{r}
\|Q(x-y)+Q(y-z)+Q(x-z)-3 Q(x)-3 Q(y)-3 Q(z)\| \\
-\|Q(x+y+z)\| \leq \lim _{m \rightarrow \infty} \frac{1}{4^{m}} \varphi\left(2^{m} x, 2^{m} y, 2^{m} z\right)=0
\end{array}
$$

for all $x, y, z \in X$. So the mapping $Q$ is quadratic.
Next, let $Q^{\prime}: X \rightarrow Y$ be another quadratic mapping satisfying (43). Then, we have

$$
\begin{aligned}
& \left\|Q(x)-Q^{\prime}(x)\right\| \\
& =\left\|\frac{1}{4^{k}} Q\left(2^{k} x\right)-\frac{1}{4^{k}} Q^{\prime}\left(2^{k} x\right)\right\| \\
& \leq \frac{1}{4^{k}}\left\{\left\|Q\left(2^{k} x\right)-f\left(2^{k} x\right)\right\|\right. \\
& \left.\quad+\left\|f\left(2^{k} x\right)-Q^{\prime}\left(2^{k} x\right)\right\|\right\} \\
& \leq \frac{1}{2} \sum_{i=0}^{\infty} \frac{1}{4^{i+k}}\left\{3 \varphi\left(2^{i+k} x,-2^{i+k} x, 0\right)\right. \\
& \left.\quad+2 \varphi\left(2^{i+k} x, 0,-2^{i+k} x\right)\right\} \\
& =\frac{1}{2} \sum_{i=k}^{\infty} \frac{1}{4^{i}}\left\{3 \varphi\left(2^{i} x,-2^{i} x, 0\right)+2 \varphi\left(2^{i} x, 0,-2^{i} x\right)\right\}
\end{aligned}
$$

for all $k \in \mathbb{N}$ and all $x \in X$. Taking the limit as $k \rightarrow \infty$, we conclude that $Q(x)=Q^{\prime}(x)$ for all $x \in X$. This completes the proof.

We obtain the following corollary concerning the stability for approximate mappings controlled by a sum of powers of norms.

Corollary 7. Let $\theta_{i} \geq 0$ be a real number and $p_{i}$ a positive real number with $p_{i}<2$ or $p_{i}>2$ for all $i=1,2$, 3. If a mapping $f: X \rightarrow Y$ with $f(0)=0$ satisfies the inequality

$$
\begin{gathered}
\|f(x-y)+f(y-z)+f(x-z)-3 f(x)-3 f(y)-3 f(z)\| \\
\leq\|f(x+y+z)\|+\theta_{1}\|x\|^{p_{1}}+\theta_{2}\|y\|^{p_{2}}+\theta_{3}\|z\|^{p_{3}}
\end{gathered}
$$

for all $x, y, z \in X$, then we can find a unique quadratic mapping $Q: X \rightarrow Y$ satisfying the inequality

$$
\begin{aligned}
\|f(x)-Q(x)\| \leq & \frac{5 \theta_{1}}{\left|4-2^{p_{1}}\right|}\|x\|^{p_{1}} \\
& +\frac{3 \theta_{2}}{\left|4-2^{p_{2}}\right|}\|x\|^{p_{2}}+\frac{2 \theta_{3}}{\left|4-2^{p_{3}}\right|}\|x\|^{p_{3}}
\end{aligned}
$$

for all $x \in X$.

\section{Stability of (5) in Non-Archimedean Spaces by Fixed Point Method}

A non-Archimedean valuation in a field $\mathbb{K}$ is a function $|\cdot|$ : $\mathbb{K} \rightarrow[0, \infty)$ with the following:

(i) $|r|=0$ if and only if $r=0$;

(ii) $|r s|=|r||s|$ for all $r, s \in \mathbb{K}$;

(iii) $|r+s| \leq \max \{|r|,|s|\}$ for all $r, s \in \mathbb{K}$. 
Any field endowed with a non-Archimedean valuation is said to be a non-Archimedean field; in any such field we have $|1|=|-1|=1$ and $|n| \leq 1$ for all $n \in \mathbb{N}$.

Definition 8. Let $\mathrm{X}$ be a linear space over a field $\mathbb{K}$ with a nonArchimedean nontrivial valuation $|\cdot|$. A function $\|\cdot\|: X \rightarrow$ $[0, \infty)$ is said to be a non-Archimedean norm if it satisfies the following conditions:

(i) $\|x\|=0$ if and only if $x=0$;

(ii) $\|r x\|=|r|\|x\|$, for all $r \in \mathbb{K}$ and $x \in X$;

(iii) $\|x+y\| \leq \max \{\|x\|,\|y\|\}$, for all $x, y \in X$.

Then $(X,\|\cdot\|)$ is called a non-Archimedean normed space.

Definition 9. Let $\left\{x_{n}\right\}$ be a sequence in a non-Archimedean normed space $X$.

(1) A sequence $\left\{x_{n}\right\}_{n=1}^{\infty}$ in a non-Archimedean space is a Cauchy sequence if the sequence $\left\{x_{n+1}-x_{n}\right\}_{n=1}^{\infty}$ converges to zero.

(2) The sequence $\left\{x_{n}\right\}$ is said to be convergent if, for any $\varepsilon>0$, there are a positive integer $N$ and $x \in X$ such that

$$
\left\|x_{n}-x\right\|<\varepsilon, \quad \forall n \geq N .
$$

Then the point $x \in X$ is called the limit of the sequence $\left\{x_{n}\right\}$, which is denoted by $\lim _{n \rightarrow \infty} x_{n}=x$.

(3) If every Cauchy sequence in $X$ converges, then the non-Archimedean normed space $X$ is called a nonArchimedean Banach space.

In 2007, Moslehian and Rassias [28] proved the generalized Hyers-Ulam stability of the Cauchy and quadratic functional equations in non-Archimedean normed spaces. Some papers $[29,30]$ on the stability of various functional equations in non-Archimedean normed spaces have been published after their stability results.

In this section, assume that $X$ is a non-Archimedean normed space and that $Y$ is a non-Archimedean Banach space. Now, we are going to investigate the stability of the functional inequality (5) in non-Archimedean Banach space by using fixed point method.

Theorem 10. Suppose that a mapping $f: X \rightarrow Y$ with $f(0)=$ 0 satisfies the functional inequality (27) and that there exists a constant $L$ with $0<L<1$ for which the perturbing function $\varphi: X^{3} \rightarrow[0, \infty)$ satisfies

$$
\begin{gathered}
\varphi(2 x, 2 y, 2 z) \leq|4| L \varphi(x, y, z) \\
\left(\varphi\left(\frac{x}{2}, \frac{y}{2}, \frac{z}{2}\right) \leq \frac{L}{|4|} \varphi(x, y, z), \text { resp. }\right)
\end{gathered}
$$

for all $x, y, z \in X$. Then, there exists a unique quadratic mapping $Q: X \rightarrow Y$ given by $Q(x)=\lim _{m \rightarrow \infty}\left(1 / 4^{m}\right) f\left(2^{m} x\right)$ $\left(Q(x)=\lim _{m \rightarrow \infty} 4^{m} f\left(x / 2^{m}\right)\right.$, resp. $)$ such that

$$
\begin{gathered}
\|f(x)-Q(x)\| \leq \frac{1}{|4|(1-L)} \Phi(x), \\
\left(\|f(x)-Q(x)\| \leq \frac{L}{|4|(1-L)} \Phi(x), \text { resp. }\right)
\end{gathered}
$$

for all $x \in X$, where $\Phi(x):=\max \{|3| \varphi(x,-x, 0),|2| \varphi(x$, $0,-x)\}$ for all $x \in X$.

Proof. From (31) and (32), we get by using the non-Archimedean norm

$$
\begin{aligned}
\|f(2 x)-4 f(x)\| & \leq \max \{|3|\|f(2 x)-2 f(x)-2 f(-x)\|, \\
& |2|\|f(2 x)-f(x)-3 f(-x)\|\} \\
& \leq \max \{|3| \varphi(x,-x, 0),|2| \varphi(x, 0,-x)\} \\
& : \equiv \Phi(x)
\end{aligned}
$$

and so

$$
\left\|f(x)-\frac{1}{4} f(2 x)\right\| \leq \frac{1}{|4|} \Phi(x)
$$

for all $x \in X$. Applying the similar argument to the corresponding proof of Theorem 4 on the complete generalized metric space $(S, d)$, we get the desired result.

We obtain the following corollary concerning the stability for approximate mappings controlled by a sum of powers of norms.

Corollary 11. Let $\theta \geq 0$ be a real number and $p$ a positive real number with $p \neq 2$. If a mapping $f: X \rightarrow Y$ with $f(0)=0$ satisfies the inequality

$$
\begin{gathered}
\|f(x-y)+f(y-z)+f(x-z)-3 f(x)-3 f(y)-3 f(z)\| \\
\leq\|f(x+y+z)\|+\theta\left(\|x\|^{p}+\|y\|^{p}+\|z\|^{p}\right)
\end{gathered}
$$

for all $x, y, z \in X$, then we can find a unique quadratic mapping $Q: X \rightarrow Y$ satisfying the inequality

$$
\|f(x)-Q(x)\| \leq \frac{\max \{|3|,|2|\}}{\left.|| 2\right|^{2}-|2|^{p} \mid} 2 \theta\|x\|^{p}
$$

for all $x \in X$.

\section{Stability of (5) in Non-Archimedean Spaces by Direct Method}

Now, we are going to investigate the stability of the functional inequality (5) in non-Archimedean Banach space by direct method. In this section, assume that $X$ is a non-Archimedean normed space and that $Y$ is a non-Archimedean Banach space. 
Theorem 12. Suppose that a mapping $f: X \rightarrow Y$ with $f(0)=$ 0 satisfies the functional inequality (27) and that $\varphi: X^{3} \rightarrow$ $[0, \infty)$ is a function such that

$$
\begin{gathered}
\lim _{m \rightarrow \infty} \frac{1}{|4|^{m}} \varphi\left(2^{m} x, 2^{m} y, 2^{m} z\right)=0, \\
\left(\lim _{m \rightarrow \infty}|4|^{m} \varphi\left(\frac{x}{2^{m}}, \frac{y}{2^{m}}, \frac{z}{2^{m}}\right)=0, \text { resp. }\right)
\end{gathered}
$$

for all $x, y, z \in X$ and

$$
\begin{gathered}
\tilde{\varphi}(x)=\lim _{m \rightarrow \infty} \max \left\{\frac{1}{|4|^{k}} \Phi\left(2^{k} x\right): 0 \leq k<m\right\} \\
\left(\tilde{\varphi}(x)=\lim _{m \rightarrow \infty} \max \left\{|4|^{k} \Phi\left(\frac{x}{2^{k}}\right): 1 \leq k<m+1\right\}, \text { resp. }\right)
\end{gathered}
$$

exists for all $x \in X$, where

$$
\Phi(x):=\max \{|3| \varphi(x,-x, 0),|2| \varphi(x, 0,-x)\}
$$

for all $x \in X$. Then there exists a quadratic mapping $Q$ : $X \rightarrow Y$ defined by $Q(x)=\lim _{m \rightarrow \infty}\left(1 / 4^{m}\right) f\left(2^{m} x\right),(Q(x)=$ $\lim _{m \rightarrow \infty} 4^{m} f\left(x / 2^{m}\right)$, resp. $)$ such that

$$
\|f(x)-Q(x)\| \leq \frac{1}{|4|} \widetilde{\varphi}(x)
$$

for all $x \in X$. Moreover, if

$$
\begin{aligned}
& \lim _{l \rightarrow \infty} \lim _{m \rightarrow \infty} \max \left\{\frac{1}{|4|^{k}} \Phi\left(2^{k} x\right): l \leq k<m+l\right\}=0, \\
& \left(\lim _{l \rightarrow \infty} \lim _{m \rightarrow \infty} \max \left\{|4|^{k} \Phi\left(\frac{x}{2^{k}}\right): l+1 \leq k<m+l+1\right\}\right. \\
& =0, \text { resp. })
\end{aligned}
$$

for all $x \in X$, then $Q$ is a unique quadratic mapping satisfying (61).

Proof. Replacing $x$ by $2^{m-1} x$ and dividing by $|4|^{m-1}$ in (55), we have

$$
\left\|\frac{f\left(2^{m-1} x\right)}{4^{m-1}}-\frac{f\left(2^{m} x\right)}{4^{m}}\right\| \leq \frac{1}{|4|} \frac{1}{|4|^{m-1}} \Phi\left(2^{m-1} x\right)
$$

for all $x \in X$. It follows from (58) and (63) that the sequence $\left\{f\left(2^{m} x\right) / 4^{m}\right\}$ is Cauchy for all $x \in X$, and the sequence $\left\{f\left(2^{m} x\right) / 4^{m}\right\}$ converges in the non-Archimedean Banach space $Y$. Therefore, we can define a mapping $Q: X \rightarrow Y$ as

$$
Q(x)=\lim _{m \rightarrow \infty} \frac{f\left(2^{m} x\right)}{4^{m}}, \quad x \in X .
$$

Applying the similar argument to the corresponding proof of Theorem 6, we get the required result.
Corollary 13. Let $\rho:[0, \infty) \rightarrow[0, \infty)$ be a function satisfying (i) $\rho(|2| t) \leq \rho(|2|) \rho(t)$ for all $t \geq 0$ and (ii) $\rho(|2|)<|2|^{2}$.

Suppose that $f: X \rightarrow Y$ with $f(0)=0$ satisfies the inequality

$$
\begin{gathered}
\|f(x-y)+f(y-z)+f(x-z)-3 f(x)-3 f(y)-3 f(z)\| \\
\leq\|f(x+y+z)\|+\varepsilon\{\rho(\|x\|)+\rho(\|y\|)+\rho(\|z\|)\}
\end{gathered}
$$

for all $x, y, z \in X$ and for some $\varepsilon>0$. Then there exists $a$ unique quadratic mapping $Q: X \rightarrow Y$ such that

$$
\|f(x)-Q(x)\| \leq \frac{1}{|4|} \max \{|3|,|2|\} 2 \varepsilon \rho(\|x\|)
$$

for all $x \in X$.

Proof. Letting $\varphi(x, y, z)=\varepsilon\{\rho(\|x\|)+\rho(\|y\|)+\rho(\|z\|)\}$, we obtain

$$
\lim _{m \rightarrow \infty} \frac{1}{|4|^{m}} \varphi\left(2^{m} x, 2^{m} y, 2^{m} z\right) \leq\left(\frac{\rho(|2|)}{|4|}\right)^{m} \varphi(x, y, z)=0
$$

for all $x, y, z \in X$. It follows from (60) that

$$
\begin{aligned}
\Phi(x) & :=\max \{|3| \varphi(x,-x, 0),|2| \varphi(x, 0,-x)\} \\
& =\max \{|3| 2 \varepsilon \rho(\|x\|),|2| 2 \varepsilon \rho(\|x\|)\} \\
& =\max \{|3|,|2|\} 2 \varepsilon \rho(\|x\|), \quad x \in X .
\end{aligned}
$$

By direct calculation,

$$
\widetilde{\varphi}(x)=\lim _{m \rightarrow \infty} \max \left\{\frac{1}{|4|^{k}} \Phi\left(2^{k} x\right): 0 \leq k<m\right\}=\Phi(x)
$$

exists and

$$
\begin{gathered}
\lim _{l \rightarrow \infty} \lim _{m \rightarrow \infty} \max \left\{\frac{1}{|4|^{k}} \Phi\left(2^{k} x\right): l \leq k<m+l\right\} \\
=\lim _{l \rightarrow \infty} \frac{1}{|4|^{l}} \Phi\left(2^{l} x\right)=0
\end{gathered}
$$

holds for all $x \in X$. Applying Theorem 12, we conclude that

$$
\begin{aligned}
\|f(x)-Q(x)\| & \leq \frac{1}{|4|} \widetilde{\varphi}(x)=\frac{1}{|4|} \Phi(x) \\
& =\frac{1}{|4|} \max \{|3|,|2|\} 2 \varepsilon \rho(\|x\|)
\end{aligned}
$$

for all $x \in X$.

Corollary 14. Let $\rho:[0, \infty) \rightarrow[0, \infty)$ be a function satisfying (i) $\rho(t /|2|) \leq \rho(1 /|2|) \rho(t)$ for all $t \geq 0$ and (ii) $\rho(1 /|2|)<$ $|2|^{-2}$. 
Suppose that a mapping $f: X \rightarrow Y$ with $f(0)=0$ satisfies the inequality

$$
\begin{aligned}
& \| f(x-y)+f(y-z)+f(x-z) \\
& -3 f(x)-3 f(y)-3 f(z) \| \\
& \quad \leq\|f(x+y+z)\|+\varepsilon\{\rho(\|x\|)+\rho(\|y\|)+\rho(\|z\|)\}
\end{aligned}
$$

for all $x, y, z \in X$ and for some $\varepsilon>0$. Then there exists a unique quadratic mapping $Q: X \rightarrow Y$ such that

$$
\begin{aligned}
\|f(x)-Q(x)\| & \leq \max \{|3|,|2|\} 2 \varepsilon \rho(\|x\|) \rho\left(\frac{1}{|2|}\right) \\
& \leq \frac{1}{|4|} \max \{|3|,|2|\} 2 \varepsilon \rho(\|x\|)
\end{aligned}
$$

for all $x \in X$.

\section{Acknowledgment}

This research was supported by the Basic Science Research Program through the National Research Foundation of Korea (NRF) funded by the Ministry of Education (no. 2012R1A1A2008139).

\section{References}

[1] S. M. Ulam, A Collection of Mathematical Problems, Interscience, New York, NY, USA, 1960.

[2] D. H. Hyers, "On the stability of the linear functional equation," Proceedings of the National Academy of Sciences of the United States of America, vol. 27, pp. 222-224, 1941.

[3] T. Aoki, "On the stability of the linear transformation in Banach spaces," Journal of the Mathematical Society of Japan, vol. 2, pp. 64-66, 1950.

[4] T. M. Rassias, "On the stability of the linear mapping in Banach spaces," Proceedings of the American Mathematical Society, vol. 72, no. 2, pp. 297-300, 1978.

[5] P. Găvruța, "A generalization of the Hyers-Ulam-Rassias stability of approximately additive mappings," Journal of Mathematical Analysis and Applications, vol. 184, no. 3, pp. 431-436, 1994.

[6] J. Aczél and J. Dhombres, Functional Equations in Several Variables, vol. 31 of Encyclopedia of Mathematics and Its Applications, Cambridge University Press, Cambridge, Mass, USA, 1989.

[7] D. H. Hyers and T. M. Rassias, "Approximate homomorphisms," Aequationes Mathematicae, vol. 44, no. 2-3, pp. 125-153, 1992.

[8] F. Skof, "Local properties and approximation of operators," Rendiconti del Seminario Matematico e Fisico di Milano, vol. 53, pp. 113-129, 1983.

[9] P. W. Cholewa, "Remarks on the stability of functional equations," Aequationes Mathematicae, vol. 27, no. 1-2, pp. 76-86, 1984.

[10] S. Czerwik, "On the stability of the quadratic mapping in normed spaces," Abhandlungen aus dem Mathematischen Seminar der Universität Hamburg, vol. 62, pp. 59-64, 1992.

[11] N. Brillouët-Belluot, J. Brzdęk, and K. Ciepliński, "On some recent developments in Ulam's type stability," Abstract and Applied Analysis, vol. 2012, Article ID 716936, 41 pages, 2012.
[12] L. Cădariu and V. Radu, "Fixed points and the stability of quadratic functional equations," Analele Universităţii de Vest din Timişoara, vol. 41, no. 1, pp. 25-48, 2003.

[13] L. C. Cădariu and V. Radu, "On the stability of the Cauchy functional equation: a fixed point approch," Grazer Mathematische Berichte, vol. 346, pp. 43-52, 2004.

[14] J. B. Diaz and B. Margolis, "A fixed point theorem of the alternative, for contractions on a generalized complete metric space," Bulletin of the American Mathematical Society, vol. 74, pp. 305-309, 1968.

[15] K. Ciepliński, "Applications of fixed point theorems to the Hyers-Ulam stability of functional equations-a survey," Annals of Functional Analysis, vol. 3, no. 1, pp. 151-164, 2012.

[16] A. Gilányi, "Eine zur Parallelogrammgleichung äquivalente Ungleichung," Aequationes Mathematicae, vol. 62, no. 3, pp. 303-309, 2001.

[17] J. Rätz, "On inequalities associated with the Jordan-von Neumann functional equation," Aequationes Mathematicae, vol. 66, no. 1-2, pp. 191-200, 2003.

[18] W. Fechner, "Stability of a functional inequality associated with the Jordan-von Neumann functional equation," Aequationes Mathematicae, vol. 71, no. 1-2, pp. 149-161, 2006.

[19] A. Gilányi, “On a problem by K. Nikodem," Mathematical Inequalities \& Applications, vol. 5, no. 4, pp. 707-710, 2002.

[20] C. Park, Y. S. Cho, and M.-H. Han, "Functional inequalities associated with Jordan-von Neumann-type additive functional equations," Journal of Inequalities \& Applications, vol. 2007, Article ID 41820, 13 pages, 2007.

[21] H.-M. Kim, K.-W. Jun, and E. Son, "Hyers-Ulam stability of Jensen functional inequality in $p$-Banach spaces," Abstract and Applied Analysis, vol. 2012, Article ID 270954, 16 pages, 2012.

[22] S.-M. Jung, Hyers-Ulam-Rassias Stability of Functional Equations in Nonlinear Analysis, vol. 48 of Springer Optimization and Its Applications, Springer, New York, NY, USA, 2011.

[23] J.-H. Bae and H.-M. Kim, "On the generalized Hyers-Ulam stability of a quadratic mapping," Far East Journal of Mathematical Sciences, vol. 3, no. 4, pp. 599-608, 2001.

[24] T. M. Rassias, "On characterizations of inner product spaces and generalizations of the $\mathrm{H}$. Bohr inequality," in Topics in Mathematical Analysis, T. M. Rassias, Ed., vol. 11, pp. 803-819, World Scientific, Singapore, 1989.

[25] C. Park, "Fixed points, inner product spaces, and functional equations," Fixed Point Theory and Applications, vol. 2010, Article ID 713675, 14 pages, 2010.

[26] S. Jang, C. Park, and H. A. Kenary, "Fixed points and fuzzy stability of functional equations related to inner product," Journal of Nonlinear Analysis and Application, vol. 2012, Article ID 00109, 15 pages, 2012.

[27] S.-M. Jung and Z.-H. Lee, "A fixed point approach to the stability of quadratic functional equation with involution," Fixed Point Theory and Applications, vol. 2008, Article ID 732086, 11 pages, 2008.

[28] M. S. Moslehian and T. M. Rassias, "Stability of functional equations in non-Archimedean spaces," Applicable Analysis and Discrete Mathematics, vol. 1, no. 2, pp. 325-334, 2007.

[29] Y. J. Cho, C. Park, and R. Saadati, "Functional inequalities in non-Archimedean Banach spaces," Applied Mathematics Letters, vol. 23, no. 10, pp. 1238-1242, 2010.

[30] J. Brzdęk and K. Ciepliński, "A fixed point theorem and the Hyers-Ulam stability in non-Archimedean spaces," Journal of Mathematical Analysis and Applications, vol. 400, no. 1, pp. 6875, 2013. 


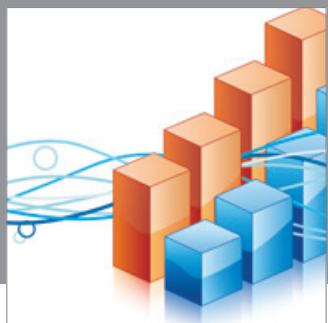

Advances in

Operations Research

mansans

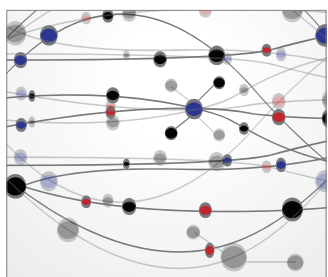

The Scientific World Journal
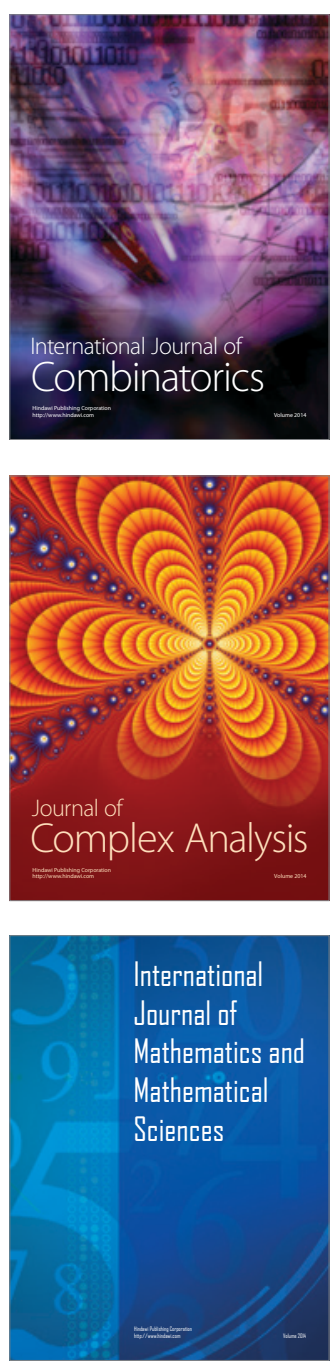
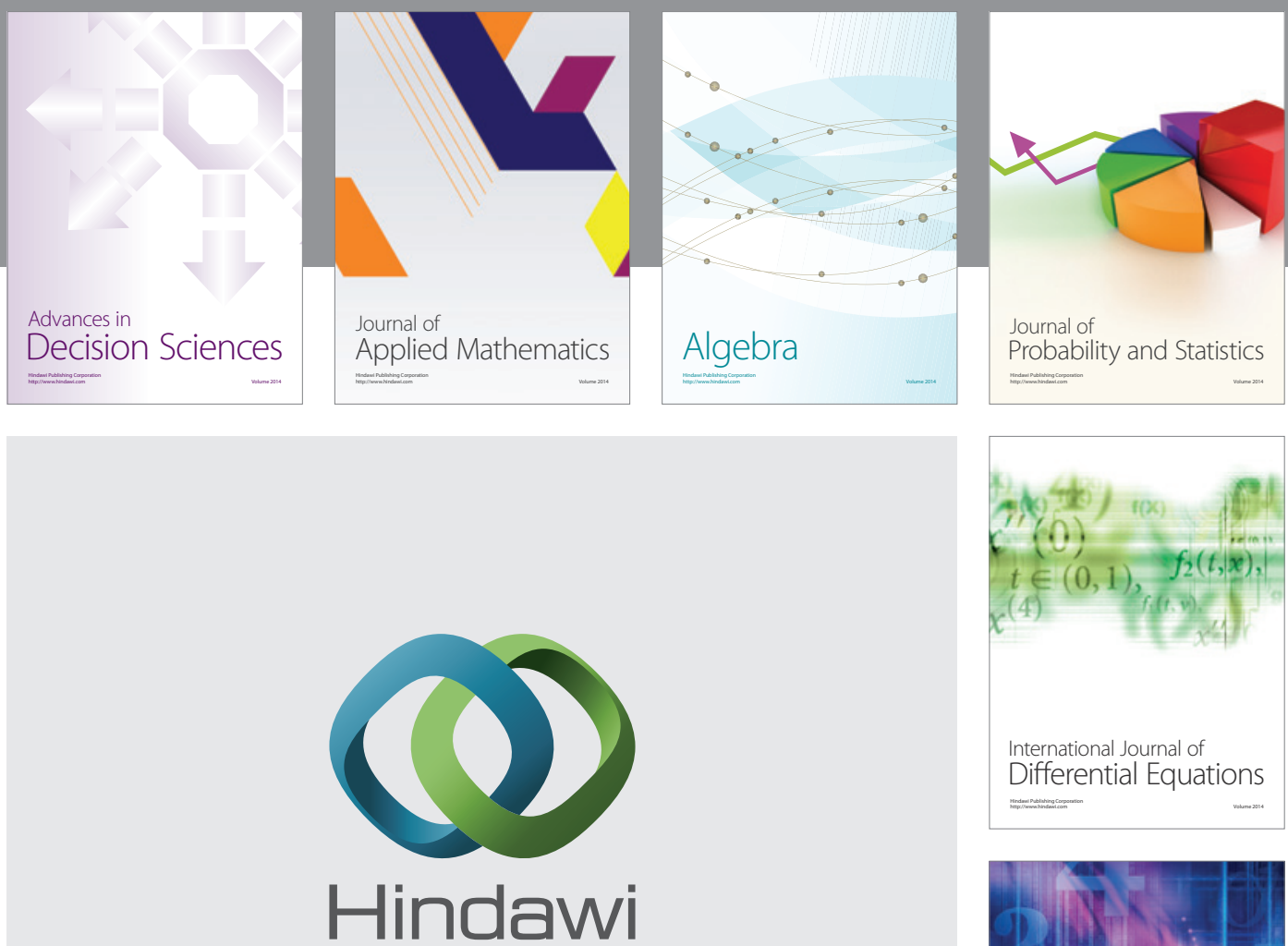

Submit your manuscripts at http://www.hindawi.com
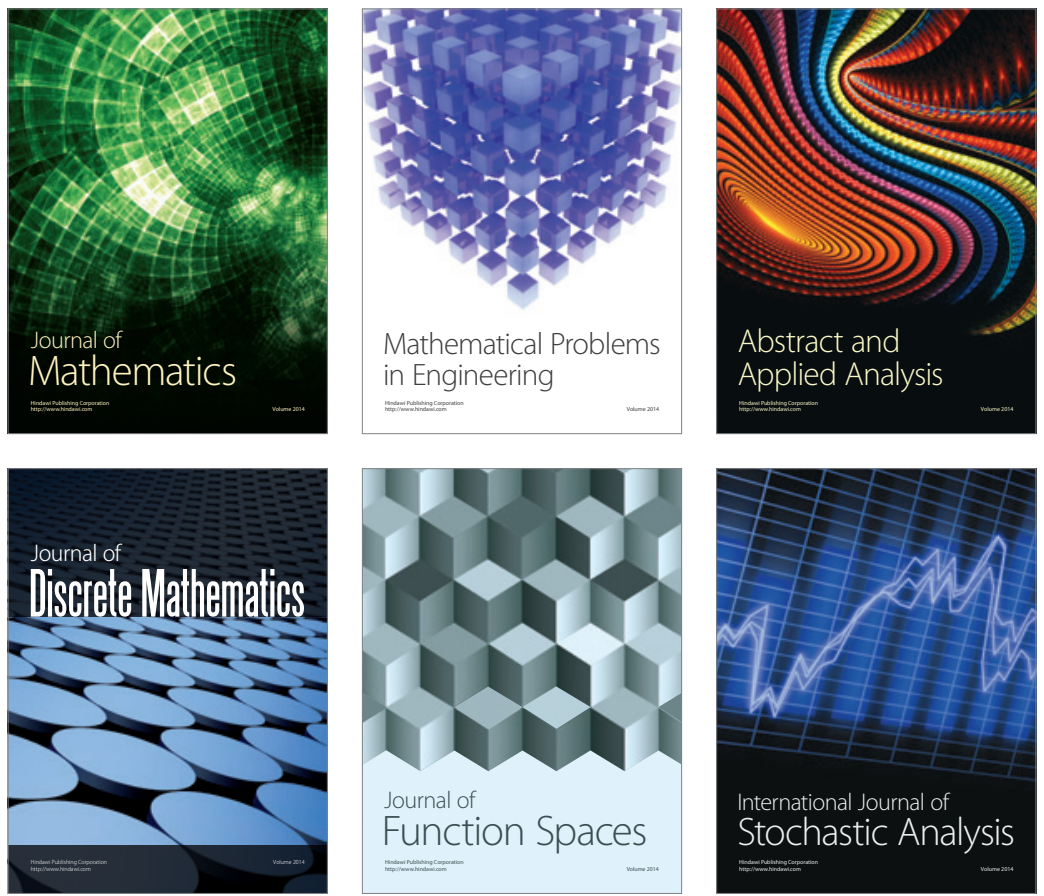

Journal of

Function Spaces

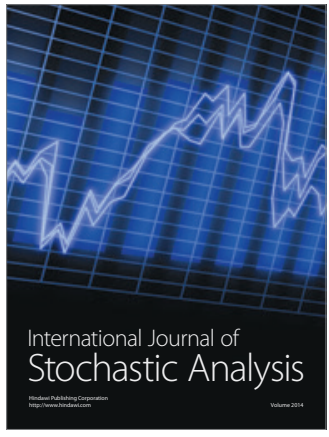

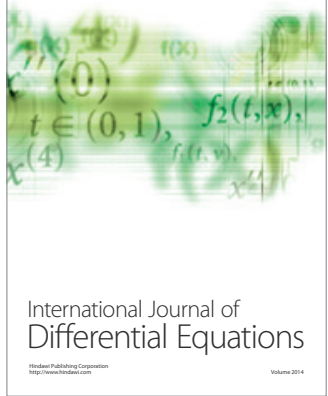
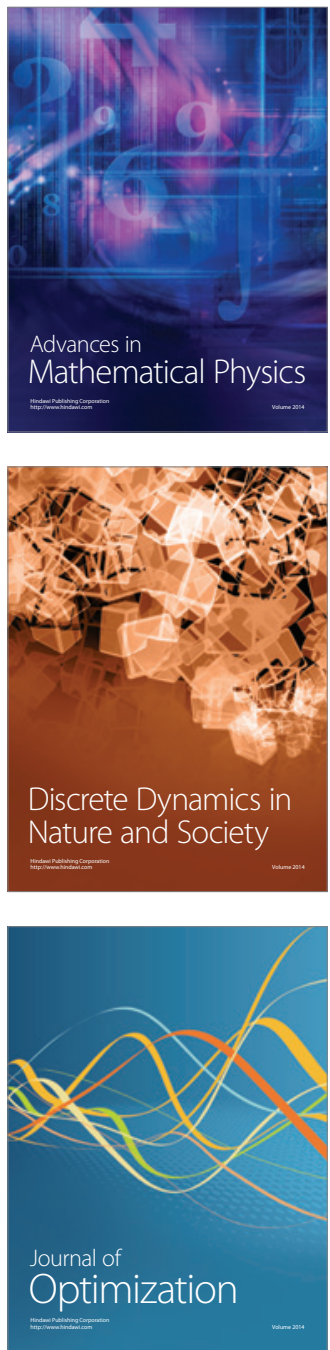